\title{
Maximum Entropy Moment Systems and Galilean Invariance
}

\author{
M. Junk* A. Unterreiter ${ }^{\dagger}$
}

\begin{abstract}
Maximum entropy moment closure systems of gas dynamics are investigated. It is shown that polynomial weight functions growing superquadratically at infinity lead to hyperbolic systems with an unpleasant state space: equilibrium states are boundary points with possibly singular fluxes. This in its generality previously unknown result applies to any moment system including, for example, the 26 or 35 moment case. One might try to avoid singular fluxes by choosing non-polynomial weight functions which grow sub-quadratically at infinity. This attempt, however, is shown to be incompatible with the Galilean invariance of the moment systems because rotational and translational invariant, finite dimensional function spaces necessarily consist of polynomials.
\end{abstract}

\section{Introduction}

Generally speaking, maximum entropy moment systems (in the sequel referred to as "MEMS") are extensions of the Euler equations of gas dynamics where non-trivial heat flux and stresses are taken into account. The derivation is based on the Boltzmann equation which models the microscopic particle movement in rarefied gases. The variables of MEMS are obtained by taking suitable weighted averages of the particle distribution functions (velocity moments). MEMS' time evolution follows from the Boltzmann equation with the additional assumption that the particle distribution function has the particular form of an entropy maximizing function whose independent variables are the involved moments. For a detailed description of this approach, we refer to [13] where several structural features of the resulting moment systems are investigated. MEMS are strictly hyperbolic because a convex entropy exists. Moreover, Galilean invariance is guaranteed if the weight functions which define the moment variables, span a translational and rotational invariant space. This condition is naturally satisfied by certain classes of polynomials which are generally used as weight functions $[7,2,15,13]$.

However, for certain model problems [8, 3, 10], polynomial weight functions of super-quadratic growth at infinity have bad side effects: The state space of the MEMS is not convex and the equilibrium states are located on the boundary.

\footnotetext{
*Universität Kaiserslautern, D-67663 Kaiserslautern, (junk@mathematik.uni-kl.de).

${ }^{\dagger}$ TU Berlin, D-10623 Berlin, (unterreiter@math.tu-berlin.de).
} 
Moreover, the flux function is possibly singular which has the surprising effect that arbitrarily close to equilibrium, arbitrarily large characteristic speeds can appear.

These results are well-known for MEMS whose weight function of highest polynomial degree is a scalar (as in the 14 or 21 moment case). As a first task in the present paper, we generalize this result as follows (Proposition 3 in Section 3): Equilibrium states are always on the boundary of the state space if polynomial weight functions of degree larger than two are used.

This result applies, e.g., to the 26 or 35 moment case.

To avoid such state spaces one may try to use non-polynomial weight functions which grow sub-quadratically at infinity.

But what are physically relevant choices for weight functions?

Due to the construction principles of MEMS in [13], we have at least to ensure that the weight functions span a rotational and translational invariant space. Otherwise the resulting MEMS is not Galilean invariant (see Section 2).

But how many finite dimensional, translational and rotational invariant spaces spanned by weight functions are there?

In Section 4, we carefully study this question to conclude: Finite dimensional, translation and rotation invariant spaces are necessarily spaces of polynomials (Theorem 11). This demonstrates the delicate relation between Galilean invariance on the one hand and a reasonable structure of the state space on the other hand: if unpleasant features of the maximum entropy systems in gas dynamics are avoided by choosing non-polynomial weight functions, Galilean invariance is lost.

The present work is closely related to previous results of T. Ruggeri [16].

Let us explain the connections in some detail.

In [16], a general class of macroscopic field equations is investigated. MEMS are included. The invariance of the field equations under the Galilean transformation $(t, \boldsymbol{x}) \rightarrow(t, \boldsymbol{x}+t \boldsymbol{u}), \boldsymbol{v} \rightarrow \boldsymbol{v}+\boldsymbol{u}$ is assumed, where $\boldsymbol{v}$ is the velocity variable of the system and $\boldsymbol{u}$ is an arbitrary vector in $\mathbb{R}^{3}$. Under these assumptions, T. Ruggeri proves a strong restriction on the possible $\boldsymbol{v}$-dependence of fluxes and productions in the equations. When applying this result to MEMS, a structural constraint for the weight functions arises. In particular, the weight functions $\boldsymbol{a}(\boldsymbol{v})=\left(a_{1}(\boldsymbol{v}), \ldots, a_{n}(\boldsymbol{v})\right)^{T}$ must be of the form (see (4.10) and (6.4) in $[16])$,

$$
\boldsymbol{a}(\boldsymbol{v})=\exp \left(\sum_{k=1}^{3} \Omega_{k} v_{k}\right) \boldsymbol{a}_{\circ}
$$

where $\Omega_{k}$ are pairwise commuting, constant $n \times n$ matrices and $\boldsymbol{a}_{\circ}$ is a vector in $\mathbb{R}^{n}$ (this is equivalent to translation invariance of $\operatorname{span}\left\{a_{1}, \ldots, a_{n}\right\}$ - see Theorem 8 in Section 4).

Assuming a certain order in the field equations, T. Ruggeri also shows that the exponential matrix of (1) has a lower triangular structure with nilpotent matrices $\Omega_{k}$. As a consequence polynomial weight functions arise.

So much for the results in [16] corresponding to our present paper. 
It is quite interesting that if one skips the assumptions leading to a lower triangular structure of the exponential matrix and to nilpotent matrices $\Omega_{k}$, formula (1) not only allows for polynomials but also for trigonometric polynomials and exponential functions. Setting, for example,

$$
\sum_{k=1}^{3} \Omega_{k} v_{k}=\left(\begin{array}{ccccccc}
0 & 0 & 0 & 0 & 0 & 0 & 0 \\
v_{1} & 0 & 0 & 0 & 0 & 0 & 0 \\
v_{2} & 0 & 0 & 0 & 0 & 0 & 0 \\
v_{3} & 0 & 0 & 0 & 0 & 0 & 0 \\
0 & v_{1} & v_{2} & v_{3} & 0 & 0 & 0 \\
0 & 0 & 0 & 0 & 0 & 0 & v_{1} \\
0 & 0 & 0 & 0 & 0 & -v_{1} & 0
\end{array}\right)
$$

we find

$$
\exp \left(\sum_{k=1}^{3} \Omega_{k} v_{k}\right)=\left(\begin{array}{ccccccc}
1 & 0 & 0 & 0 & 0 & 0 & 0 \\
v_{1} & 1 & 0 & 0 & 0 & 0 & 0 \\
v_{2} & 0 & 1 & 0 & 0 & 0 & 0 \\
v_{3} & 0 & 0 & 1 & 0 & 0 & 0 \\
|\boldsymbol{v}|^{2} / 2 & v_{1} & v_{2} & v_{3} & 1 & 0 & 0 \\
0 & 0 & 0 & 0 & 0 & \cos v_{1} & \sin v_{1} \\
0 & 0 & 0 & 0 & 0 & -\sin v_{1} & \cos v_{1}
\end{array}\right)
$$

and with $\boldsymbol{a}_{\circ}=(1,0,0,0,0,0,1)^{T}$, relation (1) yields

$$
\boldsymbol{a}(\boldsymbol{v})=\left(1, v_{1}, v_{2}, v_{3},|\boldsymbol{v}|^{2} / 2, \sin v_{1}, \cos v_{1}\right)^{T} .
$$

By construction these weight functions are translation invariant but the exponential matrix is not lower triangular.

Similarly, one can construct translation invariant systems of weight functions which contain, for example, $|\boldsymbol{v}|^{2} \sin \left(\alpha v_{i}\right) / \alpha$, where $\alpha \in \mathbb{R}^{+}$is a parameter. Taking into account that $\sin \left(\alpha v_{i}\right) / \alpha$ behaves like $v_{i}$ for small $\alpha v_{i}$, these weight functions do not grow faster than quadratically at infinity and, in a reasonable velocity range, are similar to the polynomials $|\boldsymbol{v}|^{2} v_{i}$ which are interesting because of their physical interpretation (the corresponding moments represent the energy flux). However, we will show in Section 4 that there is no possible choice of the trigonometric weight functions which gives full Galilean invariance, i.e. translation and rotation invariance.

We summarize: Galilean invariance of MEMS dictates the use of polynomials as weight functions. On the other hand polynomial weight functions with superquadratic growth at infinity lead to unpleasant properties of MEMS' state space. Hence, one either has Galilean invariance or a nice state space - but hardly ever both of them.

\section{Maximum entropy in gas dynamics}

The mathematical description of slightly rarefied gas flows requires a system of evolution equations for macroscopic quantities like mass density, flow velocity, 
temperature, heat flux, and stresses. The system should obey the basic physical properties of Galilean invariance and, in view of the second law of thermodynamics, it should satisfy a suitable $\mathrm{H}$-theorem. A standard approach to derive such a system is based on the Boltzmann equation for the distribution function $f(t, \boldsymbol{x}, \boldsymbol{v})$ of the gas particles [1]

$$
\frac{\partial f}{\partial t}+v_{j} \frac{\partial f}{\partial x_{j}}=\mathcal{C}(f), \quad \boldsymbol{x}, \boldsymbol{v} \in \mathbb{R}^{d}, t \geq 0 .
$$

Note that in (3) and in the sequel, we make use of Einstein's summation convention. We will not specify the collision operator $\mathcal{C}$ in detail but only mention those properties which are important for our investigations. Before going into details, we remark that many physically relevant quantities are obtained as velocity moments of $f$. Using $\langle\cdot, \cdot\rangle$ to denote $\boldsymbol{v}$-integration, let us consider linearly independent weight functions $a_{i}: \mathbb{R}^{d} \mapsto \mathbb{R}$ and the corresponding moments

$$
\rho_{i}(t, \boldsymbol{x})=\left\langle f(t, \boldsymbol{x}), a_{i}\right\rangle=\int_{\mathbb{R}^{d}} f(t, \boldsymbol{x}, \boldsymbol{v}) a_{i}(\boldsymbol{v}) d \boldsymbol{v}, \quad i=1, \ldots, n .
$$

As most prominent examples, we mention $a_{1}, \ldots, a_{d+2}=1, v_{1}, \ldots, v_{d},\|\boldsymbol{v}\|^{2}$ with associated macroscopic quantities

$$
\begin{aligned}
\varrho=\langle f, 1\rangle & \text { mass density } \\
\varrho \boldsymbol{u}=\langle f, \boldsymbol{v}\rangle & \text { momentum density } \\
E=\left\langle f,|\boldsymbol{v}|^{2} / 2\right\rangle & \text { energy density }
\end{aligned}
$$

Other polynomial moments represent the stress tensor and the energy flux.

In the case of a slightly rarefied gas (i.e. if the Knudsen number is a small parameter), numerical simulations of (3) are very expensive because of the high dimensionality of the problem and the stiffness of the right hand side. Since, from a physical point of view, one is rather interested in functionals of $f$ than in $f$ itself, it is a natural idea to derive equations directly for the functionals. Multiplying (3) with $\boldsymbol{a}=\left(a_{1}, \ldots, a_{n}\right)^{T}$ and integrating over $\boldsymbol{v}$, we obtain

$$
\frac{\partial \boldsymbol{\rho}}{\partial t}+\frac{\partial}{\partial x_{j}}\left\langle f, v_{j} \boldsymbol{a}\right\rangle=\langle\mathcal{C}(f), \boldsymbol{a}\rangle .
$$

The system would be closed if the particle distribution were expressed in terms of the moment vector $\rho$

$$
f(t, \boldsymbol{x}, \boldsymbol{v})=F(\boldsymbol{\rho}(t, \boldsymbol{x}), \boldsymbol{v})
$$

for some suitable $F$. A method to obtain $F$ is the maximum entropy approach where $F(\boldsymbol{\rho}, \boldsymbol{v})$ is the solution of the problem

$$
\begin{gathered}
\operatorname{maximize}-H(f) \\
\text { with } f \geq 0 \text { and }\langle f, \boldsymbol{a}\rangle=\boldsymbol{\rho}
\end{gathered}
$$

where $H(f)=\langle f \ln f-f, 1\rangle$ is a strictly convex functional (we call $H(f)$ the entropy, at variance with much of the physics literature which would denote 
it as neg-entropy). Variants and generalizations of this basic idea have been pursued by several authors (see, for example, $[4,2,6,15,13]$ ).

The formal solution of (7) is obtained with the method of Lagrange multipliers. We introduce the Lagrange functional

$$
L(f, \boldsymbol{\lambda}):=H(f)-\boldsymbol{\lambda} \cdot(\boldsymbol{\rho}-\langle f, \boldsymbol{a}\rangle)
$$

where $\boldsymbol{\lambda}$ is the vector of Lagrange multipliers. The necessary condition that all directional derivatives vanish in the maximum $f_{\boldsymbol{\lambda}}$ leads to

$$
0=\delta L\left(f_{\boldsymbol{\lambda}}, \boldsymbol{\lambda}\right)=-\ln f_{\boldsymbol{\lambda}}+\boldsymbol{\lambda} \cdot \boldsymbol{a}
$$

so that

$$
f_{\boldsymbol{\lambda}}=\exp (\boldsymbol{\lambda} \cdot \boldsymbol{a}) .
$$

In (8), the Lagrange multipliers $\boldsymbol{\lambda}$ have to satisfy the moment constraints $\boldsymbol{\rho}=$ $\left\langle f_{\boldsymbol{\lambda}}, \boldsymbol{a}\right\rangle$. It can be shown [11] that the maximum entropy problem (7) has the unique solution (8) whenever such multipliers $\boldsymbol{\lambda}=\boldsymbol{\lambda}(\boldsymbol{\rho})$ can be found. In this case, we set $F(\boldsymbol{\rho}, \boldsymbol{v})=f_{\boldsymbol{\lambda}(\boldsymbol{\rho})}(\boldsymbol{v})$.

Using the maximum entropy distribution, we can now close the moment system (6) and obtain

$$
\frac{\partial \boldsymbol{\rho}}{\partial t}+\frac{\partial}{\partial x_{j}} \boldsymbol{G}_{j}(\boldsymbol{\rho})=\boldsymbol{P}(\boldsymbol{\rho})
$$

where

$$
\boldsymbol{G}_{j}(\boldsymbol{\rho})=\left\langle F(\boldsymbol{\rho}, \boldsymbol{v}), v_{j} \boldsymbol{a}(\boldsymbol{v})\right\rangle, \quad \boldsymbol{P}(\boldsymbol{\rho})=\langle\mathcal{C}(F(\boldsymbol{\rho}, \boldsymbol{v})), \boldsymbol{a}(\boldsymbol{v})\rangle
$$

Note that the domain $\mathcal{U}$ of $\boldsymbol{G}_{j}$ and $\boldsymbol{P}$ is given by those moment vectors for which the solution $F(\boldsymbol{\rho}, \boldsymbol{v})$ of $(7)$ exists.

Now, (9) is a system of evolution equations for the relevant physical quantities and it remains to check additional properties like the existence of an entropy inequality or Galilean invariance. To discuss these topics in more detail, we first list certain properties of the collision operator $\mathcal{C}$. Following [13], we assume that $\mathcal{C}(f)$ is defined for $f \in \mathcal{D}(\mathcal{C})$, where $\mathcal{D}(\mathcal{C})$ is a set of non-negative distribution functions. Furthermore, $\mathcal{C}$ acts only on the $\boldsymbol{v}$ dependence of $f$ locally at each $(t, \boldsymbol{x})$.

The first assumption on $\mathcal{C}$ is related to dynamical conservation and can be formulated as equivalence of

$$
\begin{aligned}
& \text { i) }\langle g, \mathcal{C}(f)\rangle=0 \quad \text { for every } f \in \mathcal{D}(\mathcal{C}) \\
& \text { ii) } g \in \operatorname{span}\left\{1, v_{1}, \ldots, v_{d},|\boldsymbol{v}|^{2}\right\} .
\end{aligned}
$$

Hence, if we include the moments (5) in the vector $\boldsymbol{\rho}$, then the corresponding components of the right hand side $\boldsymbol{P}$ in (9) vanish, which means that mass, momentum, and energy are locally conserved quantities in the evolution.

Our second assumption is that $\mathcal{C}$ satisfies the dissipation relation

$$
\langle\ln f, \mathcal{C}(f)\rangle \leq 0, \quad \forall f \in \mathcal{D}(\mathcal{C})
$$


where equality is required to hold if and only if $\mathcal{C}(f)=0$ which happens exactly for the Maxwellian densities

$$
F(\varrho, \varrho \boldsymbol{u}, E, \boldsymbol{v})=\frac{\varrho}{(2 \pi \theta)^{\frac{d}{2}}} \exp \left(-\frac{|\boldsymbol{v}-\boldsymbol{u}|^{2}}{2 \theta}\right)
$$

where $\theta=\left(E / \varrho-|\boldsymbol{u}|^{2} / 2\right)$ is the rescaled temperature of the gas. Multiplying (3) with $h^{\prime}(f)$ where $h(f)=f \ln f-f$ and using (10) and mass conservation, we find that the entropy dissipates, i.e.

$$
\frac{\partial h(f)}{\partial t}+\boldsymbol{v} \cdot \nabla h(f)=\langle\ln f, \mathcal{C}(f)\rangle \leq 0 .
$$

Integrating this equation over $\boldsymbol{v}$ and introducing $H(f)=\langle h(f), 1\rangle$, we recover an entropy dissipation law (H-theorem). In [13], it is shown that this entropy dissipation law is still satisfied if we replace $f$ by the maximum entropy distribution $F(\boldsymbol{\rho})$. Hence, the physical entropy $\eta(\boldsymbol{\rho})=H(F(\boldsymbol{\rho}))$ is a locally strictly convex mathematical entropy for the system (9) which implies that (9) is symmetric hyperbolic.

Our last assumption on $\mathcal{C}$ concerns the behavior under translational and rotational transformations. Introducing for any vector $\boldsymbol{u} \in \mathbb{R}^{d}$ and any rotation matrix $R \in S O(d)$

$$
\left(\mathcal{T}_{\boldsymbol{u}} f\right)(\boldsymbol{v})=f(\boldsymbol{v}+\boldsymbol{u}), \quad\left(\mathcal{T}_{R} f\right)(\boldsymbol{v})=f(R \boldsymbol{v}), \quad \boldsymbol{v} \in \mathbb{R}^{d}
$$

the assumption on $\mathcal{C}$ reads

$$
\mathcal{T}_{\boldsymbol{u}} \mathcal{C}(f)=\mathcal{C}\left(\mathcal{T}_{\boldsymbol{u}} f\right), \quad \mathcal{T}_{R} \mathcal{C}(f)=\mathcal{C}\left(\mathcal{T}_{R} f\right) .
$$

Due to (12), the collision process is Galilean invariant and the Galilean invariance of the Boltzmann equation (3) follows, as we show now. First, we introduce the Galilean transformation $\Gamma$ which associates physical quantities in a reference space-time coordinate system $(t, \boldsymbol{x})$ to a moving system $(t, \boldsymbol{y})$. If $\boldsymbol{u} \in \mathbb{R}^{d}$ is a constant vector and $R \in S O(d)$ a fixed rotation matrix, we have the following relations for space coordinates and velocity variables

$$
\boldsymbol{y}=R \boldsymbol{x}+t \boldsymbol{u}, \quad \boldsymbol{w}=R \boldsymbol{v}+\boldsymbol{u} .
$$

We thus obtain for the distribution function $\Gamma f$ in the moving system

$$
(\Gamma f)(t, \boldsymbol{y}, \boldsymbol{w})=f\left(t, R^{T}(\boldsymbol{y}-t \boldsymbol{u}), R^{T}(\boldsymbol{w}-\boldsymbol{u})\right) .
$$

Assumption (12) together with the locality of $\mathcal{C}$ in $(t, \boldsymbol{x})$ implies $\Gamma \mathcal{C}(f)=\mathcal{C}(\Gamma f)$. Furthermore, it is a simple exercise in differentiation to show that

$$
\Gamma \frac{\partial}{\partial x_{j}}=R_{k j} \frac{\partial}{\partial y_{k}} \Gamma, \quad \Gamma \frac{\partial}{\partial t}=\frac{\partial}{\partial t} \Gamma+u_{k} \frac{\partial}{\partial y_{k}} \Gamma,
$$

and obviously, $\Gamma v_{j}=R_{k j}\left(w_{k}-u_{k}\right)$. Thus,

$$
\Gamma\left(\frac{\partial}{\partial t}+v_{j} \frac{\partial}{\partial x_{j}}-\mathcal{C}\right)=\left(\frac{\partial}{\partial t}+w_{k} \frac{\partial}{\partial y_{k}}-\mathcal{C}\right) \Gamma
$$


which shows Galilean invariance of (3).

The same property can be obtained for the corresponding moment system (9) under suitable assumptions on the weight functions $a_{i}$. Here, we adopt the sufficient condition introduced in [13] which requires that the finite dimensional function space spanned by the weight functions $a_{1}, \ldots, a_{n}$

$$
\mathbb{M}=\left\{\sum_{i=1}^{n} \beta_{i} a_{i}: \beta_{i} \in \mathbb{R}\right\}
$$

is invariant under rotations and translations, i.e.

$$
\mathcal{T}_{\boldsymbol{u}} \mathbb{M} \subset \mathbb{M}, \quad \mathcal{T}_{R} \mathbb{M} \subset \mathbb{M}, \quad \text { if } \boldsymbol{u} \in \mathbb{R}^{d}, R \in S O(d) .
$$

In other words, $\mathcal{T}_{\boldsymbol{u}} a_{i}$ and $\mathcal{T}_{R} a_{i}$ are linear combinations of the functions $a_{i}$ so that there are matrices $\Lambda(\boldsymbol{u}) \in \mathbb{R}^{n \times n}$ and $\Lambda(R) \in \mathbb{R}^{n \times n}$ such that

$$
\mathcal{T}_{\boldsymbol{u}} \boldsymbol{a}=\Lambda(\boldsymbol{u}) \boldsymbol{a}, \quad \mathcal{T}_{R} \boldsymbol{a}=\Lambda(R) \boldsymbol{a}, \quad \text { if } \boldsymbol{u} \in \mathbb{R}^{d}, R \in S O(d) .
$$

Note that $\Lambda(\boldsymbol{u}), \Lambda(R)$ are invertible with inverse $\Lambda(-\boldsymbol{u}), \Lambda\left(R^{T}\right)$. We prove

Proposition 1. Assume $F(\boldsymbol{\rho}, \boldsymbol{v})=\exp (\boldsymbol{\lambda}(\boldsymbol{\rho}) \cdot \boldsymbol{a}(\boldsymbol{v}))$ satisfies $\langle F(\boldsymbol{\rho}), \boldsymbol{a}\rangle=\boldsymbol{\rho}$. If the weight functions satisfy (15), then the moments $\Gamma \boldsymbol{\rho}=\langle\Gamma F(\boldsymbol{\rho}), \boldsymbol{a}\rangle$ in the moving system can be written as

$$
(\Gamma \boldsymbol{\rho})(t, \boldsymbol{y})=\Lambda(R) \Lambda(\boldsymbol{u}) \boldsymbol{\rho}\left(t, R^{T}(\boldsymbol{y}-t \boldsymbol{u})\right) .
$$

Moreover, we have the commutation relation

$$
\Gamma F(\boldsymbol{\rho})=F(\Gamma \boldsymbol{\rho})
$$

and the moment system (9) is Galilean invariant, i.e.

$$
\frac{\partial \Gamma \boldsymbol{\rho}}{\partial t}+\frac{\partial}{\partial y_{k}} \boldsymbol{G}_{k}(\Gamma \boldsymbol{\rho})=\boldsymbol{P}(\Gamma \boldsymbol{\rho}) .
$$

Proof. Since both translations and rotations have Jacobian determinant one, we find

$$
\Gamma \boldsymbol{\rho}=\langle\Gamma F(\boldsymbol{\rho}), \boldsymbol{a}\rangle=\left\langle\mathcal{T}_{R} \mathcal{T}_{\boldsymbol{u}} \Gamma F(\boldsymbol{\rho}), \mathcal{T}_{R} \mathcal{T}_{\boldsymbol{u}} \boldsymbol{a}\right\rangle=\Lambda(R) \Lambda(\boldsymbol{u})\left\langle\mathcal{T}_{R} \mathcal{T}_{\boldsymbol{u}} \Gamma F(\boldsymbol{\rho}), \boldsymbol{a}\right\rangle
$$

Since

$$
\left(\mathcal{T}_{R} \mathcal{T}_{\boldsymbol{u}} \Gamma F(\boldsymbol{\rho})\right)(t, \boldsymbol{y}, \boldsymbol{w})=F\left(\boldsymbol{\rho}\left(t, R^{T}(\boldsymbol{y}-t \boldsymbol{u})\right), \boldsymbol{w}\right)
$$

and $\boldsymbol{\rho}=\langle F(\boldsymbol{\rho}), \boldsymbol{a}\rangle$, we arrive at (16). Using the structure of $F(\boldsymbol{\rho}, \boldsymbol{v})=\exp (\boldsymbol{\lambda}(\boldsymbol{\rho})$. $\boldsymbol{a}(\boldsymbol{v}))$, we obtain with

$$
\boldsymbol{a}\left(R^{T}(\boldsymbol{w}-\boldsymbol{u})\right)=\left(\mathcal{T}_{R^{T}} \mathcal{T}_{-\boldsymbol{u}} \boldsymbol{a}\right)(\boldsymbol{w})=\Lambda\left(R^{T}\right) \Lambda(-\boldsymbol{u}) \boldsymbol{a}(\boldsymbol{w})
$$

that

$$
\Gamma F(\boldsymbol{\rho})=\exp \left(\left[\Lambda(-\boldsymbol{u})^{T} \Lambda\left(R^{T}\right)^{T} \boldsymbol{\lambda}(\boldsymbol{\rho})\right] \cdot \boldsymbol{a}\right) .
$$


Since $\langle\Gamma F(\boldsymbol{\rho}), \boldsymbol{a}\rangle=\Gamma \boldsymbol{\rho}$, we conclude (using uniqueness of the representation (8))

$$
\boldsymbol{\lambda}(\Gamma \boldsymbol{\rho})=\Lambda(-\boldsymbol{u})^{T} \Lambda\left(R^{T}\right)^{T} \boldsymbol{\lambda}(\boldsymbol{\rho}) .
$$

Hence, $\Gamma F(\boldsymbol{\rho})=\exp (\boldsymbol{\lambda}(\Gamma \boldsymbol{\rho}) \cdot \boldsymbol{a})=F(\Gamma \boldsymbol{\rho})$, which shows (17). Finally, Galilean invariance of the moment system is easily shown by writing (9) as

$$
\left\langle\frac{\partial F(\boldsymbol{\rho})}{\partial t}+v_{j} \frac{\partial}{\partial x_{j}} F(\boldsymbol{\rho})-\mathcal{C}(F(\boldsymbol{\rho})), \boldsymbol{a}\right\rangle=\mathbf{0} .
$$

Changing the integration variable $\boldsymbol{v}$ to $\boldsymbol{w}=R^{T}(\boldsymbol{v}-\boldsymbol{u})$ and evaluating the expression at $\boldsymbol{x}=R^{T}(\boldsymbol{y}-t \boldsymbol{u})$, we find

$$
\left\langle\Gamma\left(\frac{\partial}{\partial t}+v_{j} \frac{\partial}{\partial x_{j}}-\mathcal{C}\right) F(\boldsymbol{\rho}), \mathcal{T}_{R^{T}} \mathcal{I}_{-\boldsymbol{u}} \boldsymbol{a}\right\rangle=\mathbf{0} .
$$

Using (13) and (17), we conclude

$$
\Lambda\left(R^{T}\right) \Lambda(-\boldsymbol{u})\left\langle\left(\frac{\partial}{\partial t}+w_{k} \frac{\partial}{\partial y_{k}}-\mathcal{C}\right) F(\Gamma \boldsymbol{\rho}), \boldsymbol{a}\right\rangle=\mathbf{0}
$$

which is exactly (18) since $\Lambda\left(R^{T}\right) \Lambda(-\boldsymbol{u})$ is an invertible matrix.

\section{Polynomial weight functions}

Examples of weight functions which exhibit the invariance property are given by suitably chosen families of polynomials. Since the moment system should typically contain the conservation equations of mass, momentum, and energy, we include $\left\{1, v_{1}, \ldots, v_{d},|\boldsymbol{v}|^{2}\right\}$ in the set of weight functions. Note that the span of these polynomials is indeed invariant under rotations and translations.

By taking additional weight functions, one can generate hierarchies of symmetric hyperbolic systems which all contain the equations of mass, momentum and energy conservation but which allow, for example, a non-trivial heat flux and thus extend the classical Euler system. Following [13], we mention as examples for the three-dimensional case

$$
\begin{array}{lllllll}
10 \text { moments: } & 1 & v_{i} & |\boldsymbol{v}|^{2} / 2 & v_{i} v_{j}-|\boldsymbol{v}|^{2} \delta_{i j} & & \\
14 \text { moments: } & 1 & v_{i} & |\boldsymbol{v}|^{2} / 2 & v_{i} v_{j}-|\boldsymbol{v}|^{2} \delta_{i j} & |\boldsymbol{v}|^{2} v_{i} & |\boldsymbol{v}|^{4} \\
21 \text { moments: } & 1 & v_{i} & |\boldsymbol{v}|^{2} / 2 & v_{i} v_{j}-|\boldsymbol{v}|^{2} \delta_{i j} & v_{i} v_{j} v_{k} & |\boldsymbol{v}|^{4} \\
26 \text { moments: } & 1 & v_{i} & |\boldsymbol{v}|^{2} / 2 & v_{i} v_{j}-|\boldsymbol{v}|^{2} \delta_{i j} & v_{i} v_{j} v_{k} & |\boldsymbol{v}|^{2} v_{i} v_{j} \\
35 \text { moments: } & 1 & v_{i} & |\boldsymbol{v}|^{2} / 2 & v_{i} v_{j}-|\boldsymbol{v}|^{2} \delta_{i j} & v_{i} v_{j} v_{k} & v_{i} v_{j} v_{k} v_{l}
\end{array}
$$

Two aspects are important here: First of all, the generated function spaces are rotational and translational invariant. Furthermore, the highest degrees are always taken even to ensure that $\exp (\boldsymbol{\lambda} \cdot \boldsymbol{a}(\boldsymbol{v}))$ is integrable for a reasonable range of parameters $\boldsymbol{\lambda}$.

At first glance, the associated moment systems (9) is very promising: it generalizes the Euler equations of gas dynamics, it is strictly hyperbolic, Galilean invariant, and possesses an entropy inequality. However, a closer look reveals a fundamental problem if weight functions of fourth (or higher) order 
are used. To explain this problem, let us consider, for example, the 14-moment case. As always, the maximum entropy distribution function has the structure $F(\boldsymbol{\rho}, \boldsymbol{v})=\exp (\boldsymbol{\lambda}(\boldsymbol{\rho}) \cdot \boldsymbol{a}(\boldsymbol{v}))$, where in the 14-moment case (using Einstein's summation convention)

$$
\exp (\boldsymbol{\lambda} \cdot \boldsymbol{a}(\boldsymbol{v}))=\exp \left(\lambda+\lambda_{i} v_{i}+\lambda_{i j} v_{i} v_{j}+\bar{\lambda}_{i}|\boldsymbol{v}|^{2} v_{i}+\lambda_{14}|\boldsymbol{v}|^{4}\right) .
$$

Obviously, this distribution function is only integrable if either $\lambda_{14}<0$, or if $\lambda_{14}=0, \bar{\lambda}_{i}=0$ and $\left(\lambda_{i j}\right)$ is strictly negative definite. In the following, we denote the set of $\boldsymbol{\lambda}$ for which $\exp (\boldsymbol{\lambda} \cdot \boldsymbol{a})$ is integrable by $L$, i.e.

$$
L=\left\{\boldsymbol{\lambda} \in \mathbb{R}^{n}:\langle\exp (\boldsymbol{\lambda} \cdot \boldsymbol{a}),|\boldsymbol{a}|\rangle<\infty\right\} .
$$

The physical equilibrium states of the gas are given by Maxwellian states (11) which are of the form (20) with

$$
\lambda^{*}=\ln \frac{\varrho}{(2 \pi \theta)^{3 / 2}}-\frac{|\boldsymbol{u}|^{2}}{2 \theta}, \quad \lambda_{i}^{*}=\frac{u_{i}}{\theta}, \quad i=1,2,3, \quad \lambda_{i j}^{*}=-\frac{1}{2 \theta} \delta_{i j}
$$

and $\bar{\lambda}_{i}^{*}=\lambda_{14}^{*}=0$. These states belong to $\partial L$ because any small deviation $\boldsymbol{\lambda}$ from $\lambda^{*}$ with $\lambda_{14}>0$ does not belong to $L$. As a consequence, the equilibrium moments $\boldsymbol{\rho}^{*}$ from the set

$$
\mathcal{U}^{*}=\left\{\left\langle\varrho(2 \pi \theta)^{-d / 2} \exp \left(-|\boldsymbol{v}-\boldsymbol{u}|^{2} / 2\right), \boldsymbol{a}\right\rangle: \varrho, T>0, \boldsymbol{u} \in \mathbb{R}^{d}\right\}
$$

are also on the boundary of the state space $\mathcal{U}$

$$
\mathcal{U}=\{\langle\exp (\boldsymbol{\lambda} \cdot \boldsymbol{a}), \boldsymbol{a}\rangle: \boldsymbol{\lambda} \in L\}
$$

which is the domain of definition of equation (9). In most practical applications, there are regions in the physical space where states are close to equilibrium. Hence, $\boldsymbol{\rho}(t, \boldsymbol{x})$ touches or at least comes very close to $\partial U$ for certain $t$ and $\boldsymbol{x}$. As example, let us consider a typical Riemann initial value, consisting of two separated equilibrium states. Since the existence theory for solutions to Riemann problems with small jumps requires that the initial states are in the interior of the domain of definition and not on the boundary [17], little can be said about solvability. Also, the local existence result for smooth solutions [14] requires that the range of the initial values is contained in a compact subset of the interior of the state space [14]. Hence, initial values obtained by adding smooth and compactly supported perturbances to an equilibrium state are not covered by the local existence result if equilibrium states are located on $\partial \mathcal{U}$.

It turns out that equilibrium points at $\partial U$ is not a mere technicality. For a model problem $\left(d=1\right.$ and $\left.\boldsymbol{a}(v)=\left(1, v, v^{2}, v^{3}, v^{4}\right)\right)$, it can be shown that $\mathcal{U}$ is not convex and that the flux function $\boldsymbol{G}$ in (9) is actually singular in equilibrium points which leads to the surprising result that arbitrarily close to equilibrium, the maximal characteristic velocity of the system becomes arbitrarily large $[8$, $10,3,11]$.

We now show that equilibrium states on the boundary $\partial \mathcal{U}$ always appear when the set of weight functions contains, apart from $1, v_{i},|\boldsymbol{v}|^{2}$, other functions 
with super-quadratic growth at infinity. First, we need a general result on the solvability of moment problems for which we refer to [12, 9, 11]. Introducing the set of moments of non-negative distribution functions

$$
\mathcal{M}=\left\{\langle f, \boldsymbol{a}\rangle: f: \mathbb{R}^{d} \rightarrow \mathbb{R}^{+} \text {measurable }\right\}
$$

we have:

Theorem 2. For some $n \in \mathbb{N}$ let $a_{1}, \ldots, a_{n}$ be a set of linearly independent polynomials on $\mathbb{R}^{d}$ and assume $\boldsymbol{\rho} \in \mathbb{R}^{n}$. Then $\boldsymbol{\rho} \in \mathcal{M}$ if and only if $\boldsymbol{\beta} \cdot \boldsymbol{\rho}<0$ for all non-zero vectors $\boldsymbol{\beta} \in \mathbb{R}^{n}$ which satisfy $\boldsymbol{\beta} \cdot \boldsymbol{a}(\boldsymbol{v}) \leq 0$ for all $\boldsymbol{v} \in \mathbb{R}^{d}$.

Using this theorem, we can prove the following result.

Proposition 3. Let $n \in \mathbb{N}$ and $\mathcal{P}=\left\{a_{1}, \ldots, a_{n}\right\}$ be a set of linearly independent polynomials including $\left\{1, v_{1}, \ldots, v_{d},|\boldsymbol{v}|^{2}\right\}$. Assume that the polynomials $a_{m}, \ldots, a_{n}$ are homogeneous and of maximal degree in $\mathcal{P}$ which is larger than two and even. Then every neighborhood of an equilibrium state $\boldsymbol{\rho}^{*}$ contains moment vectors $\boldsymbol{\rho} \in \mathcal{M}$ which are not admissible states, i.e. $\boldsymbol{\rho} \notin \mathcal{U}$.

Proof. For some given $\boldsymbol{\rho}^{*} \in \mathcal{U}^{*}$, we first construct $\overline{\boldsymbol{\rho}} \in \mathcal{M}$ which is the moment vector of some non-negative density $f$. Then, we show $\overline{\boldsymbol{\rho}} \notin \mathcal{U}$ although $\left|\boldsymbol{\rho}^{*}-\overline{\boldsymbol{\rho}}\right|$ is as small as we want. For $\epsilon>0$ and $\overline{\boldsymbol{v}} \in \mathbb{R}^{d}$ with $|\overline{\boldsymbol{v}}|=1$, we set

$$
\overline{\boldsymbol{\rho}}=\boldsymbol{\rho}^{*}+\epsilon\left(0, \ldots, 0, a_{m}(\overline{\boldsymbol{v}}), \ldots, a_{n}(\overline{\boldsymbol{v}})\right) .
$$

To see that $\overline{\boldsymbol{\rho}}$ is the moment vector of some non-negative density, we use Theorem 2. If $\mathbf{0} \neq \boldsymbol{\beta} \in \mathbb{R}^{n}$ with $\boldsymbol{\beta} \cdot \boldsymbol{a}(\overline{\boldsymbol{v}}) \leq 0$ for all $\boldsymbol{v} \in \mathbb{R}^{d}$ (such a vector exists because $\mathcal{P}$ contains the constant one function) and if $2 l$ is the highest degree appearing in $\mathcal{P}$, we have

$$
0 \geq \lim _{r \rightarrow \infty} \frac{\boldsymbol{\beta} \cdot \boldsymbol{a}(r \overline{\boldsymbol{v}})}{r^{2 l}}=\sum_{i=m}^{n} \beta_{i} a_{i}(\overline{\boldsymbol{v}}),
$$

where we have used the homogeneity of $a_{m}, \ldots, a_{n}$ and the fact that $a_{1}, \ldots, a_{m-1}$ are of degree $<2 l$. Hence

$$
\boldsymbol{\beta} \cdot \overline{\boldsymbol{\rho}}=\boldsymbol{\beta} \cdot \boldsymbol{\rho}^{*}+\sum_{i=m}^{n} \beta_{i} a_{i}(\overline{\boldsymbol{v}}) \leq \boldsymbol{\beta} \cdot \boldsymbol{\rho}^{*}<0
$$

so that $\overline{\boldsymbol{\rho}} \in \mathcal{M}$ according to Theorem 2 .

We prove $\overline{\boldsymbol{\rho}} \notin \mathcal{U}$ in an indirect way. Assume $\overline{\boldsymbol{\rho}} \in \mathcal{U}$. Then there exists $\overline{\boldsymbol{\lambda}} \in L$ such that $\langle\exp (\overline{\boldsymbol{\lambda}} \cdot \boldsymbol{a}), \boldsymbol{a}\rangle=\overline{\boldsymbol{\rho}}$. Similarly, $\boldsymbol{\rho}^{*}=\left\langle\exp \left(\boldsymbol{\lambda}^{*} \cdot \boldsymbol{a}\right), \boldsymbol{a}\right\rangle$ for some $\boldsymbol{\lambda}^{*} \in L$ with $\lambda_{m}^{*}=\cdots=\lambda_{n}^{*}=0$ (because $a_{m}, \ldots, a_{n}$ are of degree $2 l>2$ and thus do not appear in equilibrium distributions). Defining

$$
\boldsymbol{\lambda}(s)=\boldsymbol{\lambda}^{*}+s\left(\overline{\boldsymbol{\lambda}}-\boldsymbol{\lambda}^{*}\right), \quad s \in[0,1]
$$

and using the convexity of the exponential function, we find an $s$-independent bound $\exp (\boldsymbol{\lambda}(s) \cdot \boldsymbol{a}) \leq \exp \left(\boldsymbol{\lambda}^{*} \cdot \boldsymbol{a}\right)+\exp (\overline{\boldsymbol{\lambda}} \cdot \boldsymbol{a})$ which suffices to show that

$$
g(s)=\langle\exp (\boldsymbol{\lambda}(s) \cdot \boldsymbol{a}), 1\rangle-\boldsymbol{\lambda}(s) \cdot \overline{\boldsymbol{\rho}}, \quad s \in[0,1]
$$


is smooth and strictly convex. Since

$$
g^{\prime}(s)=\langle\exp (\boldsymbol{\lambda}(s) \cdot \boldsymbol{a}), \boldsymbol{a}\rangle \cdot\left(\overline{\boldsymbol{\lambda}}-\boldsymbol{\lambda}^{*}\right)-\left(\overline{\boldsymbol{\lambda}}-\boldsymbol{\lambda}^{*}\right) \cdot \overline{\boldsymbol{\rho}}
$$

we conclude $g^{\prime}(1)=0$ so that $g^{\prime}(0)<0$ because $g^{\prime \prime}(s)>0$ in $[0,1]$. Hence,

$$
\begin{aligned}
0>g^{\prime}(0)=\left(\left\langle\exp \left(\boldsymbol{\lambda}^{*} \cdot \boldsymbol{a}\right), \boldsymbol{a}\right\rangle-\overline{\boldsymbol{\rho}}\right) \cdot\left(\overline{\boldsymbol{\lambda}}-\boldsymbol{\lambda}^{*}\right) & \\
& =\left(\boldsymbol{\rho}^{*}-\overline{\boldsymbol{\rho}}\right) \cdot\left(\overline{\boldsymbol{\lambda}}-\boldsymbol{\lambda}^{*}\right)=-\epsilon \sum_{i=m}^{n} \bar{\lambda}_{i} a_{i}(\overline{\boldsymbol{v}})
\end{aligned}
$$

where we have used the definition of $\bar{\rho}$ and the fact that $\lambda_{i}^{*}=0$ for $i=m, \ldots, n$. Since $a_{i}$ are continuous functions, we obtain for some suitable $\delta>0$

$$
\sum_{i=m}^{n} \bar{\lambda}_{i} a_{i}(\overline{\boldsymbol{v}}) \geq \mu>0, \quad \text { for }|\boldsymbol{v}-\overline{\boldsymbol{v}}|<\delta
$$

so that

$$
\lim _{r \rightarrow \infty} \frac{\overline{\boldsymbol{\lambda}} \cdot \boldsymbol{a}(r \boldsymbol{v})}{r^{2 l}} \geq \mu>0, \quad \text { for }|\boldsymbol{v}-\overline{\boldsymbol{v}}|<\delta .
$$

In particular, the integral over $\exp (\overline{\boldsymbol{\lambda}} \cdot \boldsymbol{a})$ is infinite because the exponential exceeds one on a domain $\{r \boldsymbol{v}: r>R,|\boldsymbol{v}-\overline{\boldsymbol{v}}|<\delta\}$ which has infinite measure. Since we have assumed $\overline{\boldsymbol{\lambda}} \in L$, i.e. $\langle\exp (\overline{\boldsymbol{\lambda}} \cdot \boldsymbol{a}), 1\rangle<\infty$, this is a contradiction. We conclude with the remark that one can generalize the definition of $\bar{\rho}$ in to

$$
\overline{\boldsymbol{\rho}}=\boldsymbol{\rho}^{*}+\epsilon \sum_{i=1}^{s} \sigma_{i}\left(0, \ldots, 0, a_{m},\left(\overline{\boldsymbol{v}}_{i}\right), \ldots, a_{n}\left(\overline{\boldsymbol{v}}_{i}\right)\right)
$$

where $\left|\boldsymbol{v}_{i}\right|=1$ and $\sigma_{i} \geq 0, \sigma_{1}>0$, without changing much of the proof.

The proof clearly shows that equilibrium points are on the boundary because weight functions are present which dominate $|\boldsymbol{v}|^{2}$ for large $|\boldsymbol{v}|$. Hence, to avoid the disadvantage $\mathcal{U}^{*} \subset \partial \mathcal{U}$, we should choose weight functions which satisfy

$$
\lim _{|\boldsymbol{v}| \rightarrow \infty} \frac{\left|a_{i}(\boldsymbol{v})\right|}{1+|\boldsymbol{v}|^{2}}=0, \quad i=2, \ldots, n
$$

where we assume $a_{1}(\boldsymbol{v})=|\boldsymbol{v}|^{2}$. Under this condition, the state space $\mathcal{U}$ coincides with the open convex set $\mathcal{M}$ of moments belonging to non-negative distribution functions [9]. In particular, equilibrium points are then always interior points which allows the application of existence results and rules out the case that the flux functions $\boldsymbol{G}_{j}$ become singular in equilibrium states.

However, the flexibility in the choice of the weight functions is restricted by the requirement of Galilean invariance for the resulting moment system. In Section 2, we have seen that the weight functions should span a rotation and translation invariant space. Therefore, we now pose the following question: Is it possible to find families of weight functions which include $a_{1}(\boldsymbol{v})=|\boldsymbol{v}|^{2}$ as well as $\left\{1, v_{1}, \ldots, v_{d}\right\}$ and give rise to rotation and translation invariant function spaces while satisfying condition (21)?

Unfortunately, the answer is no, as we prove in the next section: under suitable assumptions, a finite dimensional function space is rotation and translation invariant if and only if it is a rotation and translation invariant space of polynomials - which contradicts (21), once we want to take more weight functions than $1, v_{i}, v_{i} v_{j}$. 


\section{Invariant function spaces}

In the following, $\mathcal{F}(A, B)$ denotes the set of functions mapping the set $A$ into the set $B$. We recall that translations and rotations are introduced as linear operators on $\mathcal{F}\left(\mathbb{R}^{d}, \mathbb{R}\right)$ according to

$$
\left(\mathcal{T}_{\boldsymbol{x}} \varphi\right)(\boldsymbol{z})=\varphi(\boldsymbol{z}+\boldsymbol{x}), \quad\left(\mathcal{T}_{R} \varphi\right)(\boldsymbol{z})=\varphi(R \boldsymbol{z}), \quad \forall \boldsymbol{z} \in \mathbb{R}^{d}
$$

where $\boldsymbol{x} \in \mathbb{R}^{d}$ and $R \in S O(d)$. We put

$$
\mathcal{T}_{*}=\left\{\mathcal{T}_{\boldsymbol{x}}: \boldsymbol{x} \in \mathbb{R}^{d}\right\} \cup\left\{\mathcal{T}_{R}: R \in S O(d)\right\} .
$$

Using this notation, our aim is to characterize finite dimensional subspaces $\mathbb{M} \subset \mathcal{F}\left(\mathbb{R}^{d}, \mathbb{R}\right)$ which are invariant under the family $\mathcal{T}_{*}$. We start with some preliminary considerations.

\subsection{Generalities}

If $\mathbb{M} \subset \mathcal{F}\left(\mathbb{R}^{d}, \mathbb{R}\right)$ is finite dimensional, we can fix a basis

$$
a_{1}, \ldots, a_{n} \in \mathbb{M}
$$

where $n \in \mathbb{N}$. In the sequel we set for $\boldsymbol{z} \in \mathbb{R}^{d}$,

$$
\boldsymbol{a}(\boldsymbol{z}):=\left(a_{1}(\boldsymbol{z}), \ldots, a_{n}(\boldsymbol{z})\right)^{T} .
$$

Proposition 4. There are $\boldsymbol{z}_{1}, \ldots, \boldsymbol{z}_{n} \in \mathbb{R}^{d}$ such that $\boldsymbol{a}\left(\boldsymbol{z}_{1}\right), \ldots, \boldsymbol{a}\left(\boldsymbol{z}_{n}\right)$ are linearly independent.

Proof. It suffices to prove: $U:=\operatorname{span}\left(\left\{\boldsymbol{a}(\boldsymbol{z}): \boldsymbol{z} \in \mathbb{R}^{d}\right\}\right)=\mathbb{R}^{n}$. If $\operatorname{dim}(U)<n$, then there are $\eta_{1}, \ldots, \eta_{n} \in \mathbb{R}$ such that $\eta_{1} y_{1}+\ldots+\eta_{n} y_{n}=0$ for all $\left(y_{1}, \ldots, y_{n}\right) \in$ $U$. As a consequence, $\eta_{1} a_{1}(\boldsymbol{z})+\ldots+\eta_{n} a_{n}(\boldsymbol{z})=0$ for all $\boldsymbol{z} \in \mathbb{R}^{d}$, i.e. $a_{1}, \ldots, a_{n}$ are not linearly independent in $\mathcal{F}\left(\mathbb{R}^{d}, \mathbb{R}\right)$.

Proposition 5. Let $\Omega, \Omega_{\circ} \in \mathbb{R}^{n \times n}$ and let $\left\{\mathbf{w}_{1}, \ldots, \mathbf{w}_{n}\right\}$ be a basis of $\mathbb{R}^{n}$. Assume

$$
\forall j \in\{1, \ldots, n\}: \quad \Omega \cdot \mathbf{w}_{j}=\Omega_{\circ} \cdot \mathbf{w}_{j} .
$$

Then $\Omega=\Omega_{\circ}$.

Proof. Evident.

Corollary 1. Let $\Omega, \Omega_{\circ} \in \mathbb{R}^{n \times n}$. Assume

$$
\forall \boldsymbol{z} \in \mathbb{R}^{d}: \quad \Omega \cdot \boldsymbol{a}(\boldsymbol{z})=\Omega_{\circ} \cdot \boldsymbol{a}(\boldsymbol{z}) .
$$

Then $\Omega=\Omega_{\text {。 }}$.

Proof. Take $\boldsymbol{z}=\boldsymbol{z}_{j}, j=1, \ldots, n$ as in Proposition 4 and apply Proposition 5. 
Proposition 6. Let $\Omega \in \mathcal{F}\left(\mathbb{R}, \mathbb{R}^{n \times n}\right)$. Let $\boldsymbol{\psi} \in \mathcal{F}\left(\mathbb{R}^{d}, \mathbb{R}^{n}\right)$. Assume

$$
\forall \boldsymbol{z} \in \mathbb{R}^{d}: \quad \lim _{t \rightarrow 0} \Omega(t) \cdot \boldsymbol{a}(\boldsymbol{z})=\boldsymbol{\psi}(\boldsymbol{z}) .
$$

Then there is a matrix $\Omega_{\circ} \in \mathbb{R}^{n \times n}$ such that $\lim _{t \rightarrow 0} \Omega(t)=\Omega_{\circ}$.

Proof. We introduce for $t \in \mathbb{R}$ the matrix

$$
\Omega_{1}(t):=\Omega(t) \cdot\left(\begin{array}{ccc}
a_{1}\left(\boldsymbol{z}_{1}\right) & \ldots & a_{1}\left(\boldsymbol{z}_{n}\right) \\
\vdots & \vdots & \vdots \\
a_{n}\left(\boldsymbol{z}_{1}\right) & \ldots & a_{n}\left(\boldsymbol{z}_{n}\right)
\end{array}\right)=: \Omega(t) \cdot \mathbf{V}
$$

Due to assumption we have

$$
\lim _{t \rightarrow 0} \Omega_{1}(t)=\left(\begin{array}{ccc}
\psi_{1}\left(\boldsymbol{z}_{1}\right) & \ldots & \psi_{1}\left(\boldsymbol{z}_{n}\right) \\
\vdots & \vdots & \vdots \\
\psi_{n}\left(\boldsymbol{z}_{1}\right) & \ldots & \psi_{n}\left(\boldsymbol{z}_{n}\right)
\end{array}\right)=: \mathbf{U}
$$

Hence

$$
\lim _{t \rightarrow 0} \Omega(t)=\lim _{t \rightarrow 0} \Omega_{1}(t) \cdot \mathbf{V}^{-1}=\mathbf{U} \cdot \mathbf{V}^{-1}
$$

\subsection{Translation Invariance}

Now let $\mathbb{M}$ be an $n$-dimensional subspace of $\mathcal{F}\left(\mathbb{R}^{d}, \mathbb{R}\right)$ which is invariant under translations $\mathcal{T}_{\boldsymbol{x}}, \boldsymbol{x} \in \mathbb{R}^{d}$. Then, we can find for each $j \in\{1, \ldots, n\}$ functions $\lambda_{j, 1}, \ldots, \lambda_{j, n}: \mathbb{R}^{d} \rightarrow \mathbb{R}$ such that

$$
\mathcal{T}_{\boldsymbol{x}} a_{j}=\lambda_{j, 1}(\boldsymbol{x}) a_{1}+\ldots+\lambda_{j, n}(\boldsymbol{x}) a_{n} .
$$

We introduce the matrix-valued function

$$
\Lambda: \mathbb{R}^{d} \rightarrow \mathbb{R}^{n \times n}, \quad \Lambda(\boldsymbol{x})=\left(\lambda_{j, l}(\boldsymbol{x})\right)_{j, l=1, \ldots, n} .
$$

Then we have for all $\boldsymbol{x}, \boldsymbol{z} \in \mathbb{R}^{d}$,

$$
\boldsymbol{a}(\boldsymbol{x}+\boldsymbol{z})=\left(\begin{array}{c}
a_{1}(\boldsymbol{x}+\boldsymbol{z}) \\
\vdots \\
a_{n}(\boldsymbol{x}+\boldsymbol{z})
\end{array}\right)=\Lambda(\boldsymbol{x}) \cdot\left(\begin{array}{c}
a_{1}(\boldsymbol{z}) \\
\vdots \\
a_{n}(\boldsymbol{z})
\end{array}\right)=\Lambda(\boldsymbol{x}) \cdot \boldsymbol{a}(\boldsymbol{z}) .
$$

In particular, if we set $\boldsymbol{x}=\mathbf{0}$, then

$$
\boldsymbol{a}(\boldsymbol{z})=\Lambda(\mathbf{0}) \cdot \boldsymbol{a}(\boldsymbol{z}),
$$

hence, since $a_{1}, \ldots, a_{n}$ is a basis of $\mathbb{M}, \Lambda(\mathbf{0})=\mathrm{id}_{n \times n}$, which is the $n \times n$-identity matrix. Furthermore, if we set $\boldsymbol{z}=\mathbf{0}$, then

$$
\boldsymbol{a}(\boldsymbol{x})=\Lambda(\boldsymbol{x}) \cdot \boldsymbol{a}_{\circ},
$$


where $\boldsymbol{a}_{\circ}:=\boldsymbol{a}(\mathbf{0})$. As a consequence of (22) we have for all $\boldsymbol{x}, \boldsymbol{y}, \boldsymbol{z} \in \mathbb{R}^{d}$,

$$
\begin{aligned}
(\Lambda(\boldsymbol{x}) \cdot \Lambda(\boldsymbol{y})) \cdot \boldsymbol{a}(\boldsymbol{z}) & =\Lambda(\boldsymbol{x}) \cdot(\Lambda(\boldsymbol{y}) \cdot \boldsymbol{a}(\boldsymbol{z}))=\Lambda(\boldsymbol{x}) \cdot \boldsymbol{a}(\boldsymbol{y}+\boldsymbol{z}) \\
& =\boldsymbol{a}(\boldsymbol{x}+(\boldsymbol{y}+\boldsymbol{z}))=\boldsymbol{a}((\boldsymbol{x}+\boldsymbol{y})+\boldsymbol{z})=\Lambda(\boldsymbol{x}+\boldsymbol{y}) \cdot \boldsymbol{a}(\boldsymbol{z}),
\end{aligned}
$$

hence by Corollary 1

$$
\Lambda(\boldsymbol{x}) \cdot \Lambda(\boldsymbol{y})=\Lambda(\boldsymbol{x}+\boldsymbol{y}), \quad \forall \boldsymbol{x}, \boldsymbol{y} \in \mathbb{R}^{d} .
$$

In a similar way we deduce

$$
\Lambda(\boldsymbol{y}) \cdot \Lambda(\boldsymbol{x})=\Lambda(\boldsymbol{y}+\boldsymbol{x}), \quad \forall \boldsymbol{x}, \boldsymbol{y} \in \mathbb{R}^{d},
$$

and therefore due to $\boldsymbol{x}+\boldsymbol{y}=\boldsymbol{y}+\boldsymbol{x}$,

$$
\Lambda(\boldsymbol{x}) \cdot \Lambda(\boldsymbol{y})=\Lambda(\boldsymbol{y}) \cdot \Lambda(\boldsymbol{x})=\Lambda(\boldsymbol{x}+\boldsymbol{y}) .
$$

Now we introduce for $k \in\{1, \ldots, d\}$ the $k$-th unit vector $\boldsymbol{e}_{k}$ of $\mathbb{R}^{d}$ the oneparameter family

$$
\Lambda_{k}: \mathbb{R} \rightarrow \mathbb{R}^{n \times n}, \quad \Lambda_{k}(t)=\Lambda\left(t e_{k}\right),
$$

of matrices. Due to (22) we have for all $k \in\{1, \ldots, d\}$, for all $t \in \mathbb{R}$, and for all $z \in \mathbb{R}^{d}$

$$
\Lambda_{k}(t) \cdot \boldsymbol{a}(\boldsymbol{z})-\boldsymbol{a}(\boldsymbol{z})=\boldsymbol{a}\left(\boldsymbol{z}+t \boldsymbol{e}_{k}\right)-\boldsymbol{a}(\boldsymbol{z}),
$$

such that

$$
\lim _{t \rightarrow 0} \frac{\Lambda_{k}(t) \cdot \boldsymbol{a}(\boldsymbol{z})-\boldsymbol{a}(\boldsymbol{z})}{t}=\left(\partial_{k} \boldsymbol{a}\right)(\boldsymbol{z})
$$

if $\boldsymbol{a}$ possesses first partial derivatives in $\boldsymbol{z}$. Note that, because of translation invariance, this differentiability property follows already if we assume it in a single point.

Lemma 7. Let $\mathbb{M}=\operatorname{span}\left\{a_{1}, \ldots, a_{n}\right\}$ be translation invariant and assume that there exists $\overline{\boldsymbol{x}} \in \mathbb{R}^{d}$ where the functions $a_{1}, \ldots, a_{n}$ possess all partial derivatives $\left(\partial_{k} a_{i}\right)(\overline{\boldsymbol{x}}), i=1, \ldots, n, k=1, \ldots, d$. Then partial derivatives of $a_{i}$ exist in every point $\boldsymbol{z} \in \mathbb{R}^{d}$.

Proof. Writing

$$
\begin{array}{r}
\frac{a_{i}\left(\boldsymbol{z}+t \boldsymbol{e}_{k}\right)-a_{i}(\boldsymbol{z})}{t}=\frac{a_{i}\left(\overline{\boldsymbol{x}}+t \boldsymbol{e}_{k}+(\boldsymbol{z}-\overline{\boldsymbol{x}})\right)-a_{i}(\overline{\boldsymbol{x}}+(\boldsymbol{z}-\overline{\boldsymbol{x}}))}{t} \\
=\Lambda(\boldsymbol{z}-\overline{\boldsymbol{x}}) \cdot \frac{a_{i}\left(\overline{\boldsymbol{x}}+t \boldsymbol{e}_{k}\right)-a_{i}(\overline{\boldsymbol{x}})}{t}
\end{array}
$$

we conclude that $\left(\partial_{k} a_{i}\right)(\boldsymbol{z})=\Lambda(\boldsymbol{z}-\overline{\boldsymbol{x}})\left(\partial_{k} a_{i}\right)(\overline{\boldsymbol{x}})$.

Hence, if $\boldsymbol{a}$ has all partial derivatives in a single point, then (25) holds and with Proposition 6, we can deduce: There is for each $k \in\{1, \ldots, d\}$ a matrix $\Omega_{k} \in \mathbb{R}^{n \times n}$ such that

$$
\lim _{t \rightarrow 0} \frac{\Lambda_{k}(t)-\mathrm{id}_{n \times n}}{t}=\Omega_{k} .
$$

As a consequence, $\Lambda_{k}$ is differentiable at $t=0$ and $\Omega_{k}=\Lambda_{k}^{\prime}(0), k \in\{1, \ldots, d\}$. 
Due to (24) we have for all $s, t \in \mathbb{R}$ and for all $k \in\{1, \ldots, d\}$ the semigroup property

$$
\Lambda_{k}(s+t)=\Lambda_{k}(s) \cdot \Lambda_{k}(t)=\Lambda_{k}(t) \cdot \Lambda_{k}(s),
$$

and - naturally - $\Lambda_{k}(0)=\mathrm{id}_{n \times n}$. We deduce for all $t \in \mathbb{R}$, for all $\boldsymbol{\psi} \in \mathbb{R}^{n}$ and for all $k \in\{1, \ldots, d\}$,

$$
\begin{aligned}
\lim _{s \rightarrow 0} \frac{\Lambda_{k}(t+s) \cdot \boldsymbol{\psi}-\Lambda_{k}(t) \cdot \boldsymbol{\psi}}{s}=\lim _{s \rightarrow 0} \frac{\Lambda_{k}(s)-\mathrm{id}_{n \times n}}{s} \cdot\left(\Lambda_{k}(t) \cdot \boldsymbol{\psi}\right) & =\Omega_{k} \cdot(\Lambda(t) \cdot \boldsymbol{\psi}),
\end{aligned}
$$

i.e. if we consider the mapping $\phi():. \mathbb{R} \rightarrow \mathbb{R}^{n}, \boldsymbol{\phi}(t)=\Lambda(t) \cdot \boldsymbol{\psi}$, then $\boldsymbol{\psi}($.$) is$ differentiable (thus, continuous) and satisfies for each $t \in \mathbb{R}$ the ODE

$$
\phi^{\prime}(t)=\Omega_{k} \cdot \phi(t)
$$

subject to the initial condition

$$
\phi(0)=\psi
$$

As a consequence, we have for each $t \in \mathbb{R}$ and for each $k \in\{1, \ldots, d\}$,

$$
\phi(t)=\exp \left(t \Omega_{k}\right) \cdot \boldsymbol{\psi},
$$

and therefore due to Proposition 5,

$$
\Lambda_{k}(t)=\exp \left(t \Omega_{k}\right), \quad k \in\{1, \ldots, d\} .
$$

Due to (24) we have for all $k, l \in\{1, \ldots, d\}$ the commutator relation

$$
\left[\Lambda_{k}, \Lambda_{l}\right]=\Lambda_{k} \cdot \Lambda_{l}-\Lambda_{l} \cdot \Lambda_{k}=\mathbf{0}_{n \times n} .
$$

Hence, with (26),

$$
\left[\Omega_{k}, \Omega_{l}\right]=\mathbf{0}_{n \times n}, \quad k, l \in\{1, \ldots, d\},
$$

and therefore for all $s, t \in \mathbb{R}$ and for all $k, l \in\{1, \ldots, d\}$,

$$
\exp \left(t \Omega_{k}+s \Omega_{l}\right)=\exp \left(t \Omega_{k}\right) \cdot \exp \left(s \Omega_{l}\right)=\exp \left(s \Omega_{l}\right) \cdot \exp \left(t \Omega_{k}\right) .
$$

Thus, we have for all $\boldsymbol{x}=\left(x_{1}, \ldots, x_{d}\right) \in \mathbb{R}^{d}$,

$$
\Lambda(\boldsymbol{x})=\exp \left(\sum_{k=1}^{d} x_{k} \Omega_{k}\right), \quad\left[\Omega_{k}, \Omega_{l}\right]=\mathbf{0}_{n \times n}, \quad k, l \in\{1, \ldots, d\},
$$

and, due to (23),

$$
\boldsymbol{a}(\boldsymbol{x})=\Lambda(\boldsymbol{x}) \cdot \boldsymbol{a}_{\circ}=\exp \left(\sum_{k=1}^{d} x_{k} \Omega_{k}\right) \cdot \boldsymbol{a}_{\circ},
$$

respectively

$$
\boldsymbol{a}(\boldsymbol{x})=\exp \left(x_{1} \Omega_{1}\right) \cdot \ldots \cdot \exp \left(x_{d} \Omega_{d}\right) \cdot \boldsymbol{a}_{\circ} .
$$


Note that relation (32) drastically restricts the structure of the basis $\left\{a_{1}, \ldots, a_{n}\right\}$ of the translation invariant subspace $\mathbb{M}$ because the entries of an exponential matrix $\exp \left(x_{k} \Omega_{k}\right)$ are combinations of polynomials, exponential functions and sine and cosine functions. More precisely, if the spectrum of $\Omega_{k}$ is given by

$$
\sigma\left(\Omega_{k}\right)=\left\{\xi_{1}^{k}, \ldots, \xi_{r(k)}^{k}\right\}, \quad 1 \leq r(k) \leq n
$$

where $\xi_{w}^{k}$ are the (possibly complex) eigenvalues of $\Omega_{k}$ with respective multiplicities $\mu_{1}^{k}, \ldots, \mu_{r(k)}^{k}$, then each entry of the matrix $\exp \left(x_{k} \Omega_{k}\right)$ is the sum of terms of the form $p_{w}\left(x_{k}\right) \exp \left(x_{k} \xi_{w}^{k}\right), w=1, \ldots, r(k)$, where $p_{w}($.$) is a (complex) poly-$ nomial of degree less or equal $\mu_{w}^{k}-1$. Carrying out the matrix multiplications of (32) we obtain for all $j=1, \ldots, n$ and for all $\boldsymbol{x} \in \mathbb{R}^{d}$,

$$
\begin{aligned}
a_{j}(\boldsymbol{x}) \in \operatorname{span}_{\mathbb{C}}\left\{\boldsymbol{x}^{\boldsymbol{\alpha}} \exp (\boldsymbol{x} \cdot \boldsymbol{\xi}): \boldsymbol{\alpha}=\left(\alpha_{1}, \ldots, \alpha_{d}\right) \in \mathbb{N}_{0}^{d},\right. \\
\left.\alpha_{1}+\ldots+\alpha_{d} \leq n d, \boldsymbol{\xi}=\left(\xi_{1}, \ldots, \xi_{d}\right) \in \sigma\left(\Omega_{1}\right) \times \ldots \times \sigma\left(\Omega_{d}\right)\right\},
\end{aligned}
$$

where " $\operatorname{span}_{\mathbb{C}}\{\ldots\}$ " is the set of all complex linear combinations of elements of $\{\ldots\}$. Obviously, real eigenvalues $\xi_{w}^{k}$ give rise to exponential behavior in direction $x_{k}$ (unless $\xi_{w}^{k}=0$ which leads to a purely polynomial behavior). If an eigenvalue $\xi_{w}^{k}$ is not real, the complex conjugate is also an eigenvalue (because $\Omega_{k}$ is real) so that complex $\xi_{w}^{k}$ lead to combinations of sine and cosine functions.

We summarize our results in a final

Theorem 8. Let $\mathbb{M}$ be a translation invariant, $n$-dimensional subspace of the space $\mathcal{F}\left(\mathbb{R}^{d}, \mathbb{R}\right)$ with the property that the functions in $\mathbb{M}$ possess all partial derivatives in some point $\overline{\boldsymbol{x}} \in \mathbb{R}^{d}$. Then, all functions in $\mathbb{M}$ are analytic (combinations of polynomials, exponential functions and sine and cosine functions). More precisely, if $a_{1}, \ldots, a_{n}$ is any basis of $\mathbb{M}$, the vector $\boldsymbol{a}=\left(a_{1}, \ldots, a_{n}\right)^{T}$ can be written as

$$
\boldsymbol{a}(\boldsymbol{x})=\exp \left(\sum_{k=1}^{d} x_{k} \Omega_{k}\right) \cdot \boldsymbol{a}(\mathbf{0}), \quad \forall \boldsymbol{x} \in \mathbb{R}^{d}
$$

for certain commuting matrices $\Omega_{k} \in \mathbb{R}^{n \times n}, k=1, \ldots, d$.

\subsection{Translation Invariance and Rotation Invariance}

While translation invariance already puts a strong constraint on the subspace $\mathbb{M}$, we will now see that the additional assumption of rotation invariance further restricts the structure: the possibility that $\mathbb{M}$ contains exponential or sine and cosine functions is ruled out by rotation invariance.

We start with the observation that (using (33)): For all $R \in S O(d)$, for all $j=1, \ldots, n$ and for all $\boldsymbol{x} \in \mathbb{R}^{d}$,

$$
a_{j}(R \cdot \boldsymbol{x}) \in \operatorname{span}_{\mathbb{C}}\left\{\boldsymbol{x}^{\boldsymbol{\alpha}} \exp \left(\boldsymbol{x} \cdot\left(R^{T} \cdot \boldsymbol{\xi}\right)\right): \boldsymbol{\alpha} \in \mathbb{N}_{0}^{d},|\boldsymbol{\alpha}| \leq n d, \boldsymbol{\xi} \in \Xi\right\}
$$

where $|\boldsymbol{\alpha}|=\alpha_{1}+\ldots+\alpha_{d}$ and $\Xi=\sigma\left(\Omega_{1}\right) \times \ldots \times \sigma\left(\Omega_{d}\right)$. On the other hand, due to the assumed rotation invariance, we also have for all $R \in S O(d)$, for all 
$j=1, \ldots, n$ and for all $\boldsymbol{x} \in \mathbb{R}^{d}$,

$$
\begin{aligned}
a_{j}(R \cdot \boldsymbol{x}) \in \operatorname{span}_{\mathbb{C}}\{ & \left.a_{1}(\boldsymbol{x}), \ldots, a_{n}(\boldsymbol{x})\right\} \\
& \subseteq \operatorname{span}_{\mathbb{C}}\left\{\boldsymbol{x}^{\boldsymbol{\alpha}} \exp (\boldsymbol{x} \cdot \boldsymbol{\xi}): \boldsymbol{\alpha} \in \mathbb{N}_{0}^{d},|\boldsymbol{\alpha}| \leq n d, \boldsymbol{\xi} \in \Xi\right\} .
\end{aligned}
$$

We set for $\boldsymbol{\omega} \in \mathbb{R}^{d}$ with $|\boldsymbol{\omega}|=1$ and $t \in \mathbb{R}$,

$$
\boldsymbol{a}_{\boldsymbol{\omega}}(t):=\left(a_{\boldsymbol{\omega}}^{1}(t), \ldots, a_{\boldsymbol{\omega}}^{n}(t)\right)^{T}:=\boldsymbol{a}(t \boldsymbol{\omega}) .
$$

Then we have due to (33) for all $j \in\{1, \ldots, n\}$ and for all $t \in \mathbb{R}$,

$$
\left(a_{\boldsymbol{\omega}}^{j}\right)(t) \in \operatorname{span}_{\mathbb{C}}\left\{t^{\nu} \exp (t \boldsymbol{\omega} \cdot \boldsymbol{\xi}): 0 \leq \nu \leq n d, \boldsymbol{\xi} \in \Xi\right\}
$$

Let $\boldsymbol{\omega}^{\prime} \in \mathbb{R}^{d}$ with $\left|\boldsymbol{\omega}^{\prime}\right|=1$. Then there is $R^{\prime} \in S O(d)$ with $\boldsymbol{\omega}=R^{\prime} \cdot \boldsymbol{\omega}^{\prime}$. We deduce from (35) for all $j=1, \ldots, n$ and for all $t \in \mathbb{R}$,

$$
\left(a_{\boldsymbol{\omega}}^{j}\right)(t) \in \bigcap_{\boldsymbol{\omega}^{\prime} \in S^{2}(d)} \operatorname{span}_{\mathbb{C}}\left\{t^{\nu} \exp \left(t \boldsymbol{\omega}^{\prime} \cdot \boldsymbol{\xi}\right): 0 \leq \nu \leq n d, \boldsymbol{\xi} \in \Xi\right\},
$$

where $S^{2}(d)$ is the unit sphere in $\mathbb{R}^{d}$. Introducing

$$
\Xi^{*}:=\left(\sigma\left(\Omega_{1}\right) \backslash\{0\}\right) \times \ldots \times\left(\sigma\left(\Omega_{d}\right) \backslash\{0\}\right) .
$$

we have for all $j=1, \ldots, n$, for all $\boldsymbol{\omega} \in S^{2}(d)$ and for all $t \in \mathbb{R}$,

$$
\left(a_{\boldsymbol{\omega}}^{j}\right)(t)=p_{\boldsymbol{\omega}}(t)+\sum_{\boldsymbol{\xi} \in \Xi^{*}} p_{\boldsymbol{\omega}, \boldsymbol{\xi}}(t) \exp (t \boldsymbol{\omega} \cdot \boldsymbol{\xi}),
$$

where $p_{\boldsymbol{\omega}}(),. p_{\boldsymbol{\omega}, \boldsymbol{\xi}}$ are (possibly vanishing) polynomials in $t$ of order less or equal $n d$. We need two auxiliary results.

Proposition 9. Let $\boldsymbol{\xi}_{1}, \ldots, \boldsymbol{\xi}_{\rho} \in \mathbb{C}^{d} \backslash\{0\}, \rho \in \mathbb{N}$. Let $\boldsymbol{\omega} \in S^{2}(d)$. Then there is $\boldsymbol{\omega}^{\prime} \in S^{2}(d)$ such that

$$
\left\{\boldsymbol{\omega} \cdot \boldsymbol{\xi}_{l}: 1 \leq l \leq \rho\right\} \cap\left\{\boldsymbol{\omega}^{\prime} \cdot \boldsymbol{\xi}_{l}: 1 \leq l \leq \rho\right\}=\emptyset .
$$

Proof. We set $\alpha_{l}:=\boldsymbol{\omega} \cdot \boldsymbol{\xi}_{l}, 1 \leq l \leq \rho$. Since $\boldsymbol{\xi}_{l^{\prime}} \neq 0,1 \leq l^{\prime} \leq \rho$, the set $A_{l, l^{\prime}}:=\left\{\boldsymbol{z} \in \mathbb{R}^{d}: \boldsymbol{z} \cdot \boldsymbol{\xi}_{l^{\prime}}=\alpha_{l}\right\}$ is a hyperplane in $\mathbb{R}^{d}$. We certainly have

$$
\left\{\alpha_{l}: 1 \leq l \leq \rho\right\} \cap\left\{\boldsymbol{z} \cdot \boldsymbol{\xi}_{l}: 1 \leq l \leq \rho\right\}=\emptyset
$$

for all $z \in \mathbb{R}^{d} \backslash \bigcup_{l, l^{\prime}=1}^{\rho} A_{l, l^{\prime}}$. Since $S^{2}(d)$ is not contained in the union of finitely many hyperplanes there is $\boldsymbol{\omega}^{\prime} \in S^{2}(d) \cap\left(\mathbb{R}^{d} \backslash \bigcup_{l, l^{\prime}=1}^{\rho} A_{l, l^{\prime}}\right)$.

Proposition 10. Let $\alpha_{1}, \ldots, \alpha_{\rho} \in \mathbb{C}, \rho \in \mathbb{N}$, such that $\alpha_{l} \neq \alpha_{l^{\prime}}$ whenever $l \neq l^{\prime}$, $l, l^{\prime} \in\{1, \ldots, \rho\}$ and let $P_{1}, \ldots, P_{\rho}$ be non-vanishing complex polynomials. Then $\left\{P_{l}(t) \exp \left(\alpha_{l} t\right): 1 \leq l \leq \rho\right\}$ is a linear independent subset of $\mathcal{F}(\mathbb{R}, \mathbb{C})$. 
Proof. The functions are contained in a fundamental system of an appropriately constructed ODE system of first order with constant coefficient.

Choosing $\boldsymbol{\omega}^{\prime}$ as in Proposition 9 and ignoring vanishing terms we deduce from $(37)$

$$
\left(a_{\boldsymbol{\omega}}^{j}\right)(t)=p_{\boldsymbol{\omega}}(t)+\sum_{l=1}^{\rho} p_{\boldsymbol{\omega}, l}(t) \exp \left(t \alpha_{l}\right)=p_{\boldsymbol{\omega}^{\prime}}(t)+\sum_{l=1}^{\rho^{\prime}} p_{\boldsymbol{\omega}^{\prime}, l}(t) \exp \left(t \alpha_{\rho+l}\right),
$$

where the polynomials $p_{\boldsymbol{\omega}, 1}, \ldots, p_{\boldsymbol{\omega}, \rho}, p_{\boldsymbol{\omega}^{\prime}, 1}, \ldots, p_{\boldsymbol{\omega}^{\prime}, \rho^{\prime}}$ do not vanish (but empty sums with $\rho=0$ or $\rho^{\prime}=0$ are possible), and $\alpha_{1}, \ldots, \alpha_{\rho+\rho^{\prime}}$ are complex numbers with $\alpha_{l} \neq \alpha_{l^{\prime}}$ for $l \neq l^{\prime}, l, l^{\prime} \in\left\{1, \ldots, \rho+\rho^{\prime}\right\}$. Hence by Proposition 10: $\rho=$ $\rho^{\prime}=0$. As a consequence, $\alpha_{\boldsymbol{\omega}}^{j}$ is for each $\boldsymbol{\omega} \in S^{2}(d)$ and for each $j \in\{1, \ldots, n\}$ a polynomial of degree less or equal $n d$.

Due to (33) the function $a_{j}, j \in\{1, \ldots, n\}$, has an expansion in a Taylor series at $\boldsymbol{x}=0$. Since each $a_{\boldsymbol{\omega}}^{j}, \boldsymbol{\omega} \in S^{2}(d), j=1, \ldots, n$, is a polynomial of degree less or equal $n d$, the Taylor series of $a_{j}, j=1, \ldots, n$, has only finitely many non-vanishing terms. Thus, $a_{j}$ is for each $j=1, \ldots, n$, a polynomial, and we have shown:

Theorem 11. Let $\mathbb{M}$ be a translation and rotation invariant, finite dimensional subspace of $\mathcal{F}\left(\mathbb{R}^{d}, \mathbb{R}\right)$ with the property that the functions in $\mathbb{M}$ possess all partial derivatives in some point $\overline{\boldsymbol{x}} \in \mathbb{R}^{d}$. Then, $\mathbb{M}$ is a space of polynomials.

\section{References}

[1] C. Cercignani, The Boltzmann Equation And Its Applications, Springer, 1988 .

[2] W. Dreyer, Maximization of the entropy in non-equilibrium, J. Phys. A: Math. Gen., 20, 1987, 6505-6517.

[3] W. Dreyer, M. Junk, M. Kunik, On the approximation of kinetic equations by moment systems, Nonlinearity, 14, 2001, 881-906.

[4] B.C. Eu, A modified moment method and irreversible thermodynamics, J. Chem. Phys. 73, 1980, 2958-2969.

[5] S. Ihara, Information Theory for Continuous Systems, World Scientific, 1993.

[6] A.N. Gorban And I.V. Karlin, Method of Invariant Manifolds and the Regularization of Acoustic Spectra, Transp. Theory Stat. Phys. 23, 1994, 559-632.

[7] H. Grad, On the Kinetic Theory of Rarefied Gases, Comm. Pure \& Appl. Math. 2, 1949, 331-407.

[8] M. Junk, Domain of Definition of Levermore's Five-Moment System, J. Stat. Phys., 93, 1998, 1143-1167. 
[9] M. Junk, Maximum entropy for reduced moment problems, Math. Models Methods Appl. Sci. 10, 2000, 1001-1025.

[10] M. Junk, Maximum entropy moment problems and extended Euler equations, proceedings of IMA Workshop "Simulation of Transport in Transition Regimes", to appear

[11] M. Junk, Moment problems in kinetic theory, Habilitationsschrift, Universität Kaiserslautern, 2001.

[12] A. S. Lewis, Consistency of moment systems, Can. J. Math. 47, 1995, 995-1006.

[13] C. D. Levermore, Moment Closure Hierarchies for Kinetic Theories, J. Stat. Phys., 83, 1996, 1021-1065.

[14] A. Majda, Compressible fluid flow and systems of conservation laws in several space variables, Springer, 1984.

[15] I. Müller, T. Ruggeri, Rational Extended Thermodynamics. 2nd Edition, Springer Tracts in Natural Philosophy, Springer New York, 1998.

[16] T. RugGerI, Galilean invariance and entropy principle for systems of balance laws, Continuum Mech. Thermodyn. 1, 1989, 3-20.

[17] J. Smoller, Shock waves and reaction-diffusion equations, Springer, 1983. 